\title{
¿Es tiempo de exigir publicidad basada en la evidencia?
}

\author{
Salvador Israel Macías-Hernández y Juan Daniel Morones-Alba²
}

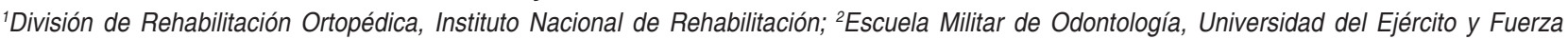
Aérea Mexicana. Ciudad de México, México

\section{Resumen}

Actualmente existe información publicitaria sobre productos médicos que no necesariamente está fundada en datos científicamente comprobados, y en paralelo se exige a los clínicos que se apeguen a guías de práctica basadas en la evidencia y que se sometan a certificaciones periódicas; este hecho resulta contradictorio. Es necesario comenzar a reflexionar sobre la necesidad de que se regule la información presentada en los medios y se antepongan el bienestar y la seguridad de los pacientes a intereses externos, que la comunidad médica exija regulaciones más estrictas y que esto derive en una publicidad basada en la evidencia.

PALABRAS CLAVE: Publicidad al consumidor. Medicina basada en la evidencia. Medios de comunicación.

\begin{abstract}
Claims made in current advertising for medical products is not necessarily scientifically proven, yet at the same time clinicians are required to adopt evidence-based practices and undergo periodic certifications. This is a clear contradiction. It is crucial to begin to reflect on the need to regulate information presented in the media and to place greater emphasis on patient well-being and safety instead of on third-party interests. The medical community must demand stricter regulations and evidence-based advertising policies.
\end{abstract}

KEY WORDS: Consumer advertising. Evidence based medicine. Communications media

En los últimos años, la publicidad de productos relacionados con la salud se ha convertido en una vorágine de anuncios promocionales que llenan a los consumidores de información sobre las características, la fisiopatología y el tratamiento de las enfermedades. Muchas de estas patologías son altamente prevalentes, discapacitantes y consideradas problemas de salud pública en México y en el mundo, e implican costos millonarios para los sistemas de salud, como la diabetes, la obesidad o la osteoartritis, que son causas importantes de morbimortalidad'.

Paralelamente existen sociedades, asociaciones e instituciones médicas nacionales e internacionales que se dedican a difundir documentos basados en la evidencia. Los documentos incluyen guías de práctica clínica, recomendaciones basadas en la evidencia, extensas revisiones de la literatura, exhaustivos metaanálisis y grandes ensayos clínicos controlados, que muestran objetivamente y bajo un rigor científico y metodológico estricto los efectos de los distintos tratamientos. Las guías y recomendaciones comprometen al clínico a ejercer su práctica de acuerdo con los principios del conocimiento científico. Asimismo, los médicos se someten a evaluaciones periódicas por consejos que certifican su competencia para ejercer la profesión.

\section{Correspondencia: \\ Salvador Israel Macías-Hernández \\ Calzada México-Xochimilco 289 \\ Col. Arenal de Guadalupe, Del. Tlalpan \\ C.P. 14389, Ciudad de México, México \\ E-mail: simacias@inr.gob.mx, \\ drisraelmacias@gmail.com}

Fecha de recepción en versión modificada: 06-01-2017

Fecha de aceptación: 11-01-2017

DOI://dx.doi.org/10.24875/GMM.17002834
Gac Med Mex. 2017;153:907-908

Contents available at PubMed www.gacetamedicademexico.com 
Resulta paradójico que, por un lado, la información de los medios de comunicación sea cada vez más vasta y superficial, que se utilicen estrategias de mercadotecnia para enfatizar los «beneficios» de los productos y se invite a consumirlos por sus efectos curativos, modificadores de la enfermedad o de alivio sintomático, no necesariamente demostrados científicamente, sin considerar las potenciales reacciones adversas o riesgos de utilizarlos, y que por otro lado se exija a los médicos que se apeguen a la práctica basada en la evidencia.

Diariamente se publican miles de estudios científicos sobre procedimientos, intervenciones, sustancias, fármacos o suplementos. Por ello, paralelo al acelerado avance de la medicina y las recomendaciones basadas en la evidencia, los medios de comunicación masiva continúan difundiendo información basada solo en estrategias de mercadotecnia. Así, por citar un ejemplo, en un estudio realizado en Australia en el que se evaluó la evidencia científica de la información en las revistas de divulgación de salud, se encontró que solo el $36 \%$ de la información presentada estaba sustentada científicamente ${ }^{2}$.

Es evidente que los intereses económicos se anteponen a las mejores prácticas clínicas, ya que muchas de las afirmaciones que se realizan en los anuncios publicitarios carecen de sustento científico ${ }^{3}$.

Desafortunadamente, existen en el mercado publicitario, por mencionar algunos, productos para el control del peso; «modificadores de la progresión de la enfermedad o regeneradores» en enfermedades como la osteoartritis; sustancias «naturales» contra síntomas de depresión; sustancias que controlan la diabetes y sus complicaciones; y analgésicos y antiinflamatorios de venta libre, que no consideran estados mórbidos de los pacientes, como gastropatías, cardiopatías o hipertensión, cuyos efectos podrían no solo ser ineficaces, sino potencialmente graves 0 incluso mortales ${ }^{4}$. Existen casos de falta de regulación gubernamental en los cuales algunos productos «milagro" son aprobados sin sustento científico para el tratamiento de enfermedades como el cáncer ${ }^{5}$.

Existe una clara y evidente necesidad de regular la publicidad médica, la cual debe ser sustentada invariablemente en evidencia científica de adecuada calidad e indudable confiabilidad, y que se anteponga el bienestar y los intereses de los pacientes a los de cualquier otro.

\section{Bibliografía}

1. Lozano R, Naghavi M, Foreman K, et al. Global and regional mortality from 235 causes of death for 20 age groups in 1990 and 2010: a systematic analysis for the Global Burden of Disease Study 2010. Lancet. 2012;380:2095-128

2. Wilson A, Smith D, Peel R, et al. A quantitative analysis of the quality and content of the health advice in popular Australian magazines. Aust N Z J Public Health. 2017:41:256-8.

3. Gale DA. The focus of the media is medical intervention not the pursuit of health. PLoS Med. 2005;2:e362.

4. Schwitzer G, Mudur G, Henry D, et al. What are the roles and responsibilities of the media in disseminating health information? PLOS Med. 2005;27:e215.

5. Kuchenbecker RS, Mota DM. Miracle drug: Brazil approves never-tested cancer medicine. J Oncol Pharm Pract. 2017;23:399-400. 